\title{
ANÁLISIS
}

\section{GESTIÓN DE CONTENIDOS CON DRUPAL: REVISIÓN DE MÓDULOS ESPECÍFICOS PARA BIBLIOTECAS, ARCHIVOS Y MUSEOS}

\section{Jesús Tramullas}

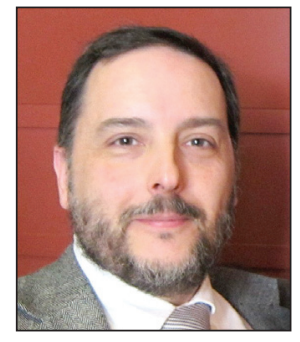

Jesús Tramullas es profesor titular en el Departamento de Ciencias de la Documentación de la Universidad de Zaragoza. Socio senior de ACM e IEEE; ISKO, Anabad y Asociación Española de Recuperación de Información (SERI). Miembro de Drupal Association. Es investigador principal de proyectos de investigación financiados sobre web semántica, web social y servicios de información digital, y miembro del grupo de investigación Intelligent Networks and Information Technologies (iNiT), reconocido por el Gobierno de Aragón. Sus líneas de investigación se centran en el diseño e implementación de servicios de información digital, gestión de contenidos, comportamiento informacional, bibliotecas digitales y herramientas de software libre para la gestión de información. http://orcid.org/0000-0002-5374-9993

Universidad de Zaragoza, Departamento de Ciencias de la Documentación Pedro Cerbuna, 12. 50009 Zaragoza, España http://tramullas.com tramullas@unizar.es

\section{Resumen}

Drupal es una de las plataformas más avanzadas de gestión de contenidos. Para la elaboración de productos especializados, la arquitectura de Drupal utiliza módulos que añaden funciones específicas a su núcleo. Existe más de una veintena de módulos orientados a las necesidades de bibliotecas, archivos y museos. Se revisan las características de esos módulos, incluyendo evolución temporal, número de versiones, desarrolladores implicados, instalaciones y otros datos.

\section{Palabras clave}

Drupal, Módulos, Gestión de contenidos, Bibliotecas, Repositorios, Opacs.

Title: Content management with Drupal: a review of modules specific to libraries, archives and museums

\begin{abstract}
Drupal is one of the most advanced platforms for content management. For product development, Drupal architecture uses modules that add specific functionality to its core. More than $\mathbf{2 0}$ modules are geared to the needs of libraries, archives and museums. This paper reviews the characteristics and development of these modules, including changes over time, number of versions, developers involved, active implementations, and other data.
\end{abstract}

\section{Keywords}

Drupal, Modules, Content management, Repositories, Libraries, Opacs.

Tramullas, Jesús (2013). "Gestión de contenidos con Drupal: revisión de módulos específicos para bibliotecas, archivos y museos". El profesional de la información, septiembre-octubre, v. 22, n. 5, pp. 425-431.

\section{Planteamiento, objetivo y método}

Drupal se ha convertido en los últimos años en el sistema de gestión de contenidos (content management system, CMS) que mayor expansión ha alcanzado entre la comunidad de diseñadores de productos y servicios del sector de bibliotecas. Su arquitectura modular y su capacidad para implementar proyectos y funciones ex-profeso, así como el creciente número de programadores especializados con que cuenta, han hecho posible la creación de todo tipo de productos y servicios basados en Drupal. El valor añadido que ofrece no ha pasado desapercibido en el ámbito de las unidades de información (Farkas, 2008; Coombs, 2009; Becker, 2010). http://drupal.org 


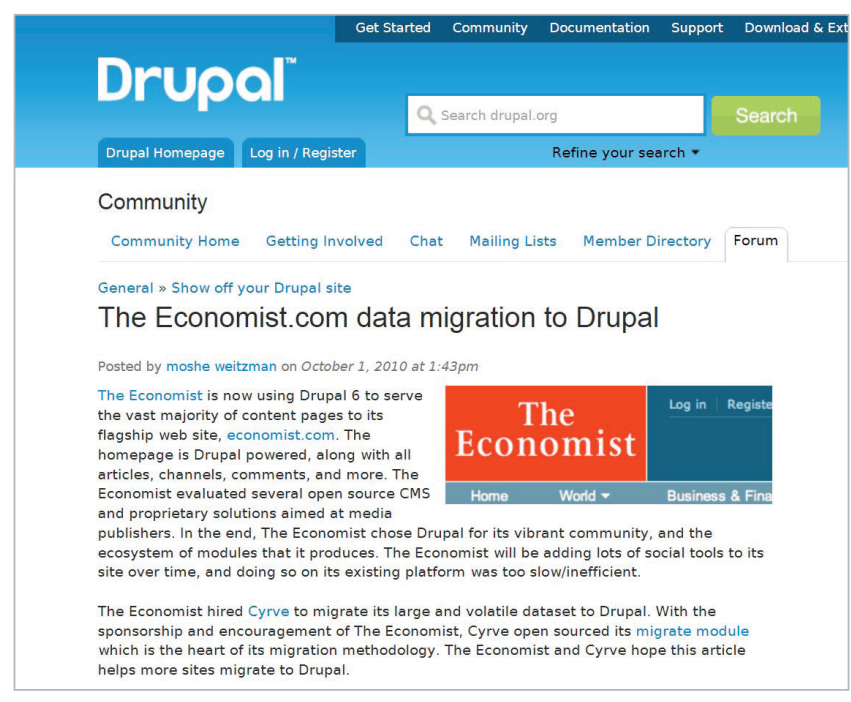

El semanario británico The economist se pasó a Drupal en octubre de 2010. Tiene 3 millones de usuarios registrados y mensualmente contabiliza 25 millones de páginas vistas.

https://drupal.org/node/915102

Sin embargo en numerosas ocasiones se acude a Drupal esperando encontrar una solución directa que resuelva los problemas y necesidades de un servicio o producto determinado. Adoptar este enfoque es un error. La incorporación de funciones específicas se realiza mediante la instalación de módulos que complementan y amplían las prestaciones que ofrece su core o núcleo (Vacek et al., 2010). Drupal se ha transformado en una plataforma sobre la cual es necesario implementar elementos específicos que ofrezcan una solución a los requerimientos planteados en la fase de diseño del producto o servicio. Este cambio, que se inició en la versión 6 , se ha consolidado en la versión 7 , y la futura versión 8 completa esta migración hacia una plataforma de desarrollo. De ser un CMS que ofrece funciones avanzadas, se está transformando en un entorno basado en un framework PHP, como es Symfony. Drupal se ha ido separando progresivamente del enfoque adoptado por productos como WordPress o Joomla, orientados a la solución directa y casi inmediata de necesidades.

http://symfony.com

Este trabajo tiene como objetivo revisar el estado de evolución de los módulos de Drupal especialmente diseñados para implementar productos y servicios de información específicos de bibliotecas, archivos y museos, de forma que se pueda transformar en un opac o portal bibliotecario (Rosenthal; Bernardo, 2010), un gestor de referencias, un servidor OAI, una biblioteca digital (Moore, 2008) o un frontend para colecciones digitales. Para ello se ha establecido el siguiente método de trabajo:

- definición de las categorías de análisis, atendiendo a las funciones, evolución y uso de los módulos;

- identificación de los módulos especialmente diseñados para bibliotecas, archivos y museos;

- revisión de los objetivos, características, versiones, modificaciones y uso de los mismos;

- discusión de resultados y elaboración de recomendaciones.
Los módulos que tiene en cuenta este trabajo han sido seleccionados tomando como referencia las enumeraciones disponibles en Library Modules, en los archivos de la lista de correo electrónico Drupal4Lib, y en Tramullas y GarridoPicazo (2010). La recopilación se ha completado mediante búsquedas específicas por descriptor/título/módulo relacionado en drupal.org. Los datos de referencia de cada uno de

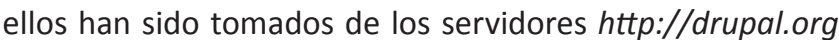
y http://drupalmodules.com, disponibles a 31 de mayo de 2013. Dado que entre los objetivos del trabajo se encuentra ofrecer un indicador sobre el desarrollo de cada módulo, se han tomado en consideración las estadísticas básicas, el número de versiones liberadas, y las aportaciones o commits. El indicador de instalaciones activas se basa en la recopilación automática de datos que hace Drupal en sus versiones 6 y 7 merced al módulo Update status. http://groups.drupal.org/libraries/modules http://listserv.uic.edu/archives/drupal4lib.html https://drupal.org/node/329620

No se han cubierto los módulos orientados a la gestión de documentos, dado que su campo de aplicación excede el marco del trabajo planteado, y porque las características difieren entre los módulos que podrían tomarse en consideración. Por el momento no existe una solución a la gestión de documentos o records management sobre Drupal, basada en la implementación de un módulo o conjunto de módulos especializados. Esta limitación no quiere decir que no sea posible implementar una gestión de documentos o una biblioteca digital sobre Drupal, a través del uso de módulos no especialmente identificados y orientados a bibliotecas, archivos y museos.

\section{Drupal en bibliotecas, archivos y museos}

2006 Se crea una web de referencia, Drupalib, actualmente con poca actividad

2006 Se crea el grupo Libraries dentro de los grupos de Drupal, que continúa activo http://groups.drupal.org/libraries

2007 Se inicia la lista de correo Drupal4Lib, que es el principal medio de intercambio de información entre desarrolladores y usuarios de Drupal en bibliotecas: http://listserv.uic.edu/archives/drupal4lib.html

2008 Austin y Harris publican Drupal in libraries, dentro de la colección Library technology reports de la $A L A$, un manual introductorio que cubría Drupal en su versión 6

2010 American libraries, la revista web de la American Library Association, adopta Drupal como su plataforma web, titulando la noticia "Drupal: the change we need" (Fitzpatrick, 2010)

2010 Se publica un volumen en el que se recogen experiencias de implementación sobre Drupal 6 en el contexto español (Tramullas, 2010). Coombs y HoIlister (2010) dedican un capítulo a Drupal dentro de su manual sobre software libre para bibliotecas

2012 ALA TechSource y Neal-Schuman publican un nuevo manual de introducción, también titulado Drupal in libraries (Varnum, 2012), que se centra en la versión 7 
La importancia de Drupal en el campo de las bibliotecas es creciente, como se muestra en la siguiente secuencia cronológica:

Tramullas y Garrido-Picazo (2010) realizaron una recopilación de módulos para bibliotecas y archivos, disponibles para Drupal 6: gestión bibliográfica, formato MARC, Z39.50, OAI, e integración en Drupal de opacs, incluyendo Millenium integration y Sopac2. A finales de 2011, el Multimedia Information Technology Group de Cilip realizó una breve enumeración de módulos de Drupal de interés para bibliotecas, bajo el título "Battle for the CMSs: Drupal for libraries" (MMIT, 2010). En él se incluían algunos módulos para Drupal 6 que no han tenido mantenimiento posterior, y señalaba sólo a Sopac y eXtensible Catalog como integradores para opacs.
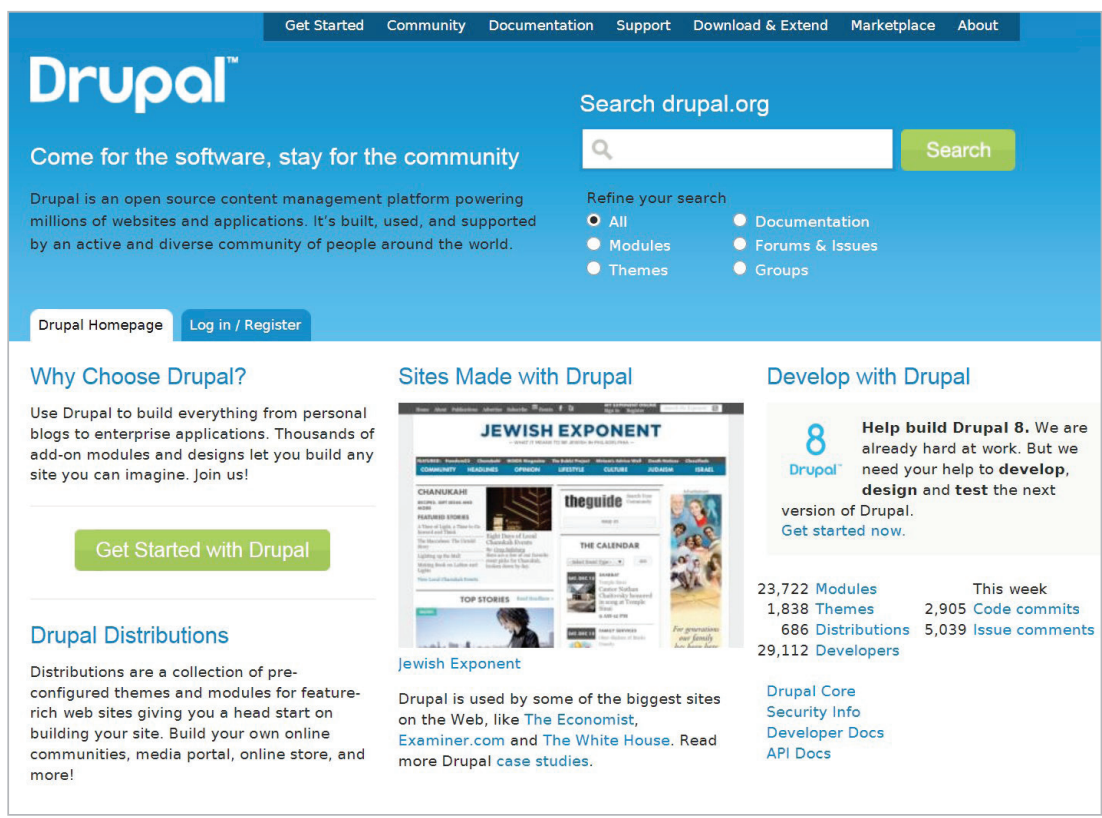

En la parte inferior derecha pueden verse las estadísticas de uso y actividad, que son buscables. https://drupal.org

\section{La incorporación de funciones específi- cas se realiza mediante la instalación de módulos que complementan y amplían las prestaciones que ofrece el núcleo de Drupal}

Frente a la gran actividad que despliegan los usuarios de bibliotecas, su penetración en archivos y museos parece limitada. Las particularidades de las colecciones digitalizadas de ambos tipos de unidades, tanto en esquemas de metadatos como en formatos, así como la existencia de herramientas especializadas para su gestión, muchas de ellas libres o gratuitas, han limitado el uso de Drupal en estos sectores. A ello cabe añadir que en los últimos años el proceso de publicación de colecciones en internet se ha beneficiado de herramientas específicas, como Collective access u Omeka. Para los usuarios de museos existe el grupo Museums, con poca actividad. Esto contrasta con el interés que genera en la comunidad museística: en las últimas ediciones de la conferencia anual Museums and the Web siempre ha habido sesiones, talleres o presentaciones dedicadas a usos de Drupal en los museos. En lo que concierne a los archivos, no existe un grupo específico de usuarios de Drupal, pero al igual que en el caso de los museos, es posible rastrear en la bibliografía y en internet e identificar proyectos de archivos que lo utilizan como plataforma.

http://collectiveaccess.org

http://www.omeka.org

https://groups.drupal.org/museums

http://www.museumsandtheweb.com

\section{Módulos para bibliotecas, archivos y museos}

Se han identificado y revisado 30 módulos (recogidos en la tabla 1). Los datos básicos han sido tomados de los servidores de referencia indicados, y han sido completados y elaborados para identificar otros hitos significativos (fechas de primeras versiones, commits, resolución de bugs, etc.). La información obtenida ha sido elaborada y organizada en fichas de detalle para cada uno de los módulos, que a su vez se han integrado en una única tabla para facilitar la visualización y la comparación.

Para su estudio se han agrupado en las siguientes categorías:

- integración con repositorio (3);

- integración con gestores de colecciones (1);

- integración de opac (6);

- gestión de ejemplares y circulación (1);

- integración de metadatos bibliográficos (6);

- autentificación de usuarios (1);

- recuperación de información/metabuscador (4);

- gestión de bibliografías (1);

- proveedor/recolector OAI (3);

- esquemas de clasificación (2);

- integración de colecciones de museos (2).

Un resumen de los datos básicos obtenidos se refleja en la tabla 2.

Frente a la gran actividad en bibliotecas, la penetración de Drupal en archivos y museos parece limitada

En lo que concierne a la publicación de código, sólo Fedora commons API, a pesar de haber sido registrado, no ha producido ni liberado ningún código. De los 29 restantes, 8 no están elaborando nuevas versiones, y de éstos sólo 2 señalan disponer de mantenimiento. Esto indica que los módulos realmente implementables en producción son 21.

Si se atiende a la disponibilidad para versiones $(5,6$ y 7$)$, Drupal 6 es la versión con mayor disponibilidad de módulos (26), de los cuales 15 han incorporado ya código adaptado a Drupal 7. A éstos hay que sumar otros 5 , que han sido publicados directamente para la versión 7 , sin código para 
Tabla 1. Módulos identificados y revisados

\begin{tabular}{|c|c|c|}
\hline Nombre & Tipo & Descripción breve del módulo \\
\hline Fedora commons API & Integración con repositorio & API para acceder y manipular contenido de repositorios Fedora commons desde Drupal \\
\hline Islandora & Integración con repositorio & Paquete completo que integra el contenido de repositorios Fedora commons en Drupal \\
\hline DSpace & Integración con repositorio & API para incorporar contenido de repositorios DSpace \\
\hline Contentdm integration & $\begin{array}{l}\text { Integración con gestores de } \\
\text { colecciones }\end{array}$ & Conjunto de módulos que permiten el acceso a colecciones depositadas en Contentdm \\
\hline $\begin{array}{l}\text { Millenium opac inte- } \\
\text { gration }\end{array}$ & Integración de opac & Integra el contenido del opac de sigb Millenium con Drupal \\
\hline Opac & Integración de opac & $\begin{array}{l}\begin{array}{l}\text { Integra el contenido del opac de diferentes sigbs, para lo que requiere la programación de } \\
\text { conectores }\end{array} \\
\end{array}$ \\
\hline PMB Drupal & Integración de opac & Proyecto experimental para integrar contenido del opac de PMB en Drupal. \\
\hline PMB connector & Integración de opac & Integra el contenido del opac de $P M B$ \\
\hline Sopac2 the social opac & Integración de opac & Integra el contenido del opac de diferentes sigbs, para lo que requiere conectores \\
\hline $\begin{array}{l}\text { eXtensible catalog } \\
\text { Drupal toolkit }\end{array}$ & Integración de opac & $\begin{array}{l}\text { Solución completa para integrar un opac o un conjunto de ellos, en Drupal. Usa recolección } \\
\text { OAI-PMH, estandariza los datos desde MARC } 21 \text { y Dublin core, y puede transformarlos en } \\
\text { FRBR y RDA. Puede incluir información en tiempo real sobre préstamo }\end{array}$ \\
\hline MARC & $\begin{array}{l}\text { Integración de metadatos } \\
\text { bibliográficos }\end{array}$ & Importa registros en formato MARC en nodos de Drupal \\
\hline Library & $\begin{array}{l}\text { Gestión de ejemplares y } \\
\text { circulación }\end{array}$ & Sirve para crear una biblioteca básica de elementos y asignar acciones sobre ellos \\
\hline LT4L & $\begin{array}{l}\text { Integración de metadatos } \\
\text { bibliográficos }\end{array}$ & Muestra datos de libros de un opac, que se asocian a un nodo de Drupal, usando su etiquetado \\
\hline Google books & $\begin{array}{l}\text { Integración de metadatos } \\
\text { bibliográficos }\end{array}$ & Inserta en nodos los datos de libros existentes en Google books \\
\hline Open library API & $\begin{array}{l}\text { Integración de metadatos } \\
\text { bibliográficos }\end{array}$ & Recupera datos de libros existentes en Open library \\
\hline Book post & $\begin{array}{l}\text { Integración de metadatos } \\
\text { bibliográficos }\end{array}$ & $\begin{array}{l}\text { Captura datos de diferentes servidores de libros (Open library, WorldCat, LibraryThing y } \\
\text { Google books), y los muestra en nodos }\end{array}$ \\
\hline ISBN2node & $\begin{array}{l}\text { Integración de metadatos } \\
\text { bibliográficos }\end{array}$ & Crea nodos con datos de libros, usando como referencia el ISBN \\
\hline EZProxy & Autentificación de usuarios. & $\begin{array}{l}\text { Implementa autentificación para usuarios de Drupal contra servidores EZProxy, para poder } \\
\text { acceder a recursos externos bajo licencia }\end{array}$ \\
\hline Z39.50/SRU client & $\begin{array}{l}\text { Recuperación de informa- } \\
\text { ción/metabuscador }\end{array}$ & Cliente Z39.50 \\
\hline Article discovery & $\begin{array}{l}\text { Recuperación de informa- } \\
\text { ción/metabuscador }\end{array}$ & Interfaz de consulta en Drupal contra servidores Summon \\
\hline $\begin{array}{l}\text { Pazpar2 metasearch } \\
\text { integration }\end{array}$ & $\begin{array}{l}\text { Recuperación de informa- } \\
\text { ción/metabuscador }\end{array}$ & Integra en Drupal el metabuscador Pazpar2 de Index Data \\
\hline Bibliography module & Gestión de bibliografías & Permite implantar un sistema web para gestión de referencias bibliográficas \\
\hline Views OAI-PMH & Proveedor/recolector OAI & Implementa un servidor OAI \\
\hline OAI2 for CCK & Proveedor/recolector OAI & Implementa un servidor OAI \\
\hline $\begin{array}{l}\text { Feeds OAl-PMH Fetcher/ } \\
\text { Parser }\end{array}$ & Proveedor/recolector OAl & Recolecta datos de servidores OAl \\
\hline Hilcc taxonomy tag & Esquema de clasificación & Asigna términos de clasificación a los nodos atendiendo a los $L C C$ call numbers \\
\hline MeSH vocabulary & Esquema de clasificación & Integra los términos del MeSH como taxonomía \\
\hline $\begin{array}{l}\text { Apache Solr search } \\
\text { integration }\end{array}$ & $\begin{array}{l}\begin{array}{l}\text { Recuperación de informa- } \\
\text { ción/metabuscador }\end{array} \\
\end{array}$ & Integra la indización y búsqueda con Solr en implementaciones de Drupal \\
\hline Collective access & $\begin{array}{l}\text { Integración de colecciones } \\
\text { de museos }\end{array}$ & Integra datos de colecciones gestionadas con Collective access \\
\hline Adlibapi & $\begin{array}{l}\text { Integración de colecciones } \\
\text { de museos }\end{array}$ & Integra datos de colecciones gestionadas con Adlib \\
\hline
\end{tabular}

las previas, lo que eleva la disponibilidad hasta los 20. Diez módulos han detenido su desarrollo en la versión 6, y no parece que vayan a liberar nuevo código adaptado a la 7 o al futuro Drupal 8. De todo el conjunto, sólo 8 módulos estaban disponibles para Drupal 5.

\section{Discusión de resultados}

No se han valorado las funciones y prestaciones que cada uno de los módulos puede ofrecer en un contexto dado, ya que no se pretende analizar la calidad del software. La información recopilada se utiliza para delinear el panorama del desarrollo y disponibilidad de los módulos para la gestión de información en unidades y servicios de información.

La primera cuestión que cabe señalar es la ausencia de módulos orientados a la integración con sistemas de gestión de archivos. Esto se debe precisamente a la capacidad de llevar a cabo cualquier tipo de contenido y la estructura de datos co- 
rrespondiente con módulos de uso general, y al potencial de importación de casi cualquier contenido que ofrece el módulo Feeds, ya señalado. Con Drupal es posible implementar procesos de gestión de documentos (records management) usando la gran variedad de módulos disponibles. Además el desarrollo de módulos que usan el estándar CMIS hace posible importar y compartir información con herramientas como Alfresco, Nuxeo, KnowledgeTree o SharePoint.

El panorama de los módulos para museos es similar. Sólo se encuentran módulos de integración de contenidos para dos sistemas de gestión de colecciones de museos (Collective access y Adlib), ya que se está usando la misma aproximación que en archivos para la generación de productos basados en Drupal.

Los repositorios disponen de mayor número de opciones. Hay dos grupos de módulos:

- los que son interfaces directas a software específico, como los módulos para DSpace o Fedora (Islandora);

- los que implementan un servidor o un recolector OAI, con el estándar Dublin core, y son capaces de ofrecer o de importar la información propia a terceros, o de recolectar registros externos e importar la información en tipos de nodos, y se apoyan en la funcionalidad del módulo de uso general Feeds.

Un tercio de los módulos revisados no ha publicado versión para Drupal 7, lo que podría plantear problemas para el mantenimiento de los servicios a corto plazo

Las bibliotecas disponen del mayor número de posibilidades. En primer lugar, cabe destacar los módulos que proveen de una capa de interfaz contra el opac de los sigbs tradicionales: Millenium (Garza, 2009), PMB, opac, eXtensible catalog... Las instalaciones no son muy numerosas, lo que puede deberse a los requerimientos y restricciones que impone a las bibliotecas el tener que acometer una implementación nueva, cuando ya se dispone de un opac en funcionamiento. Las ventajas que ofrece la integración de una capa social y de funciones de valor añadido en el opac parecen pesar menos que el potencial coste asociado. A ello cabe añadir las mejoras que han tenido los opacs en las últimas versiones de los productos comerciales.

Un segundo grupo lo formarían las herramientas para metabuscadores, como Article discovery y Pazpar2 metasearch integration (mkdru), que proveen de acceso a recursos Z39.50 y similares.

En un tercer grupo entrarían los módulos capaces de capturar información bibliográfica, y de integrarla y mostrarla en nodos de Drupal. Los datos tomados de LibraryThing, The open library o Google books pueden incorporarse a cualquier tipo de producto o servicio, que no tiene porque ser estrictamente bibliotecario. Contrasta el uso de los módulos de datos bibliográficos con la carencia de módulos orientados a importar o gestionar esquemas de clasificación, lo que puede deberse precisamente a la importación directa de los esquemas desde los catálogos, o a la utilización del módulo de uso genérico Taxonomy import/export via $X M L$, que es capaz de integrar en las taxonomías de Drupal todo tipo de esquemas de clasificación, y que muestra un elevado uso (2.371 instalaciones activas).

Una cuestión clave para la implementación de los módulos es la disponibilidad de un soporte adecuado para los problemas que puedan surgir. Dadas las características de las herramientas y de la comunidad, es necesario que los autores de código documenten adecuadamente no sólo éste, sino los procesos de instalación, configuración y ejecución del mismo. En el conjunto revisado sólo catorce módulos ofrecen documentación de referencia, lo que puede suponer un problema en determinados contextos.

La revisión de la disponibilidad y del desarrollo de módulos específicos para bibliotecas, archivos y museos en Drupal refleja la pequeña comunidad de usuarios implicados activamente en este campo

En contraste con los módulos anteriores deben destacarse, por sus características de desarrollo y utilización, Bibliography y Apache Solr search integration. El módulo Biblio es un clásico de Drupal, que lleva en producción desde la versión 4.7 en 2006, e indica tener más de 4.500 instalaciones activas. A su alrededor se ha generado un buen conjunto de 
Tabla 2. Datos básicos de cada módulo

\begin{tabular}{|c|c|c|c|c|c|c|c|c|c|}
\hline Módulo & $\begin{array}{l}\text { Núm. } \\
\text { versión }\end{array}$ & Primera & Última & D5 & D6 & D7 & $\begin{array}{l}\text { Mant. } \\
\text { activo }\end{array}$ & $\begin{array}{l}\text { Estado } \\
\text { desarr. }\end{array}$ & $\begin{array}{c}\text { Documen- } \\
\text { tación }\end{array}$ \\
\hline Fedora commons API & 0 & & & No & No & No & No & Inact. & No \\
\hline Islandora & 8 & $02 / 12 / 08$ & $18 / 03 / 13$ & No & Sí & Sí & Sí & Activo & Sí \\
\hline DSpace & 1 & $01 / 01 / 12$ & $13 / 09 / 12$ & No & No & Sí & Sí & Activo & No \\
\hline Contentdm integration & 1 & $22 / 07 / 11$ & $26 / 06 / 12$ & No & Sí & No & Sí & Activo & No \\
\hline Millenium opac integration & 17 & $15 / 01 / 08$ & $12 / 02 / 13$ & Sí & Sí & Sí & Sí & Activo & Sí \\
\hline opac & 13 & $25 / 07 / 12$ & $31 / 05 / 13$ & No & No & Sí & Sí & Activo & Sí \\
\hline PMB Drupal & 1 & $16 / 11 / 11$ & $16 / 11 / 11$ & No & Sí & No & Sí & Activo & Sí \\
\hline PMB connector & 8 & $12 / 11 / 11$ & $25 / 05 / 13$ & No & Sí & Sí & Sí & Activo & Sí \\
\hline Sopac2 the social opac & 4 & $17 / 03 / 09$ & $08 / 03 / 10$ & No & Sí & No & No & Inact. & Sí \\
\hline eXtensible catalog Drupal toolkit & 20 & $31 / 03 / 11$ & $22 / 05 / 13$ & No & Sí & Sí & Sí & Activo & Sí \\
\hline MARC & 7 & 19/12/07 & $16 / 06 / 10$ & Sí & Sí & No & No & Inact. & No \\
\hline Library & 3 & 09/08/08 & $02 / 03 / 13$ & No & Sí & Sí & No & Inact. & No \\
\hline LT4L & 4 & $14 / 10 / 10$ & $13 / 06 / 11$ & No & Sí & Sí & Sí & Activo & No \\
\hline Google books & 2 & $06 / 03 / 12$ & $24 / 02 / 13$ & No & No & Sí & Sí & Activo & Sí \\
\hline Open library API & 1 & $11 / 08 / 09$ & $25 / 05 / 13$ & No & Sí & No & No & Inact. & No \\
\hline Book post & 1 & 07/10/08 & 07/10/08 & NO & Sí & No & No & Inact. & No \\
\hline ISBN2node & 3 & 07/06/11 & $09 / 06 / 11$ & No & No & Sí & Sí & Activo & No \\
\hline EZProxy & 9 & 28/04/09 & 05/01/11 & Sí & Sí & Sí & Sí & Activo & Sí \\
\hline Z39.50/SRU client & 4 & $01 / 05 / 06$ & 25/07/09 & Sí & Sí & No & No & Inact. & No \\
\hline Article discovery & 1 & $07 / 04 / 11$ & $07 / 04 / 11$ & No & Sí & Sí & No & Activo & No \\
\hline Pazpar2 metasearch integration & 5 & $02 / 08 / 11$ & $02 / 04 / 13$ & No & Sí & Sí & Sí & Activo & Sí \\
\hline Bibliography module & 89 & $23 / 06 / 06$ & $31 / 05 / 13$ & Sí & Sí & Sí & Sí & Activo & Sí \\
\hline Views OAI-PMH & 8 & $30 / 05 / 11$ & $21 / 05 / 13$ & No & Sí & Sí & Sí & Activo & No \\
\hline OAI2 for $C C K$ & 5 & 06/08/08 & $23 / 11 / 11$ & Sí & Sí & No & Sí & Inact. & No \\
\hline Feeds OAI-PMH Fetcher/Parser & 3 & $17 / 10 / 10$ & 06/09/11 & No & Sí & Sí & Sí & Activo & Sí \\
\hline HILCC taxonomy tag & 2 & $12 / 03 / 09$ & $25 / 02 / 11$ & Sí & Sí & No & Sí & Activo & No \\
\hline MeSH vocabulary & 4 & $11 / 01 / 11$ & $11 / 01 / 11$ & No & Sí & No & Sí & Inact. & No \\
\hline Apache Solr search integration & 86 & $25 / 12 / 07$ & $26 / 05 / 13$ & Sí & Sí & Sí & Sí & Activo & Sí \\
\hline Collective access & 8 & $14 / 08 / 11$ & $02 / 02 / 12$ & No & Sí & Sí & Sí & Activo & Sí \\
\hline Adlibapi & 2 & $09 / 02 / 13$ & $09 / 02 / 13$ & No & No & Sí & Sí & Activo & No \\
\hline
\end{tabular}

submódulos que amplían sus funciones, hasta configurar un completo sistema de gestión bibliográfica y de referencias. En su desarrollo se han generado casi 2.800 commits (aportaciones), por parte de 17 desarrolladores. La integración de Solr como motor de búsqueda para propósitos múltiples en Drupal es exitosa; su capacidad para trabajar con información estructurada en $\mathrm{xml}$ le da especial interés para las unidades de información. Varios de los módulos analizados incluyen una implementación de Solr. Al igual que Biblio, muestra un patrón de comunidad masivo: en cinco años, 2.100 commits y 53 desarrolladores.

Los datos de comunidad y desarrollo de Biblio y Solr sirven como contrapunto para el resto de módulos. La mayoría de los analizados indican uno o dos desarrolladores, con un número de commits bajo. Sólo Millenium opac integration, opac y extensible catalog muestran una pauta activa de commits, aunque Millenium sólo tiene 1 desarrollador. Esta pauta indica que el desarrollo de la mayoría de los módulos tiende a ser unipersonal, con poca o nula comunidad impli- cada, y que depende de encargos específicos o de interés personal. Esta tendencia es un riesgo que puede afectar la evolución del producto, y amenazar la aparición de nuevas versiones o actualizaciones. Una segunda pauta es la que marcan empresas de software libre, que aplican el módulo como uno más de los productos que ofrecen a sus clientes, como opac, Pazpar2 metasearch integration ( $m k d r u$ ) y PMB connector. Relacionado con lo anterior está el bajo número de versiones del módulo que se suele publicar (en el recuento se han considerado tanto las versiones estables como las de desarrollo), excepción hecha de los cinco módulos señalados en este párrafo. También cabe señalar que el módulo puede dejar de actualizarse mientras cumpla adecuadamente con la finalidad para la cual fue diseñado, lo que también parece suceder en varios casos de los analizados.

La evolución de las versiones de Drupal también tiene su incidencia en los módulos. Un tercio de los revisados no ha publicado versión para Drupal 7, lo que podría plantear problemas para el mantenimiento de los servicios a corto plazo, cuando 
se publique la versión 8 , y se deje de dar soporte a la versión 6. La mayor parte de estos módulos publicaron su última versión hace dos o más años, y, aunque señalan encontrarse mantenidos y en producción, cabe dudar de la fiabilidad de alguno de estos datos. Sin embargo, varios tienen sus funciones cubiertas por nuevos módulos para la versión 7, o por actualizaciones de otros módulos existentes, que han ampliado sus funciones.

\section{Conclusiones}

La revisión de la disponibilidad de módulos específicos para bibliotecas, archivos y museos en Drupal refleja la pequeña comunidad de usuarios implicados activamente en este campo. En los próximos años, el diseño e implementación de productos y servicios más avanzados va a hacer necesario que bibliotecas, archivos y museos se planteen la necesidad de dedicar medios a la implementación de software que responda específicamente a sus necesidades. Esto deberá ser llevado cabo en colaboración, mediante la asunción o dotación compartida de recursos.

La implementación de estas funciones resulta en ocasiones compleja para un usuario de tipo medio, como suele ser el profesional del sector. En ello inciden factores como la evaluación de funcionamiento de los módulos, la compatibilidad entre versiones, las opciones de instalación y/o configuración, etc. Están apareciendo distribuciones o perfiles de instalación, que simplifican los procesos necesarios para disponer de una instalación plenamente operativa. Por ejemplo, se encuentran disponibles eXtensible Catalog (XC) Installation, OAIConnect installation profile, o AgriDrupal (Subirats et al., 2010). https://drupal.org/project/xc_installation https://drupal.org/project/oaiconnect

Drupal es una de las plataformas de gestión de contenidos más extendida y con mayor funcionalidad, publicada bajo licencia de software libre. En el mercado puede encontrarse un elevado número de profesionales y empresas que ofrecen soporte y desarrollo. El modelo de negocio aplicado, basado en el ofrecimiento de valor añadido, funciona correctamente, como demuestra también el ejemplo de WordPress. Drupal es por tanto una base sólida para la implementación de diferentes tipos de productos o servicios de información digital.

\section{Bibliografía}

Austin, Andy; Harris, Christopher (2008). Drupal in libraries. Library technology reports, v. 44, n. 4, Chicago: ALA. http://dx.doi.org/10.5860/Itr.44n4

Becker, Danielle A. (2010). "Adventures in Drupal. Designing a library website using a CMS". Online, v. 34, n. 5, pp. 19-21.

Coombs, Karen (2009). "Drupal done right”. Library journal, v. 134, n. 19 , pp. 30-32.

Coombs, Karen; Hollister, Amanda (2010). Open source web applications for libraries. Information today. ISBN: 978 1573874007

Farkas, Meredith (2008). “CMS for next-gen websites: using Drupal to manage library web content". American libraries, v. 39, n. 10.

Fitzpatrick, Sean (2010). "Drupal: the change we need". American libraries.

http://www.americanlibrariesmagazine.org/blog/drupalchange-we-need

Garza, Alejandro (2009). "From OPAC to CMS: Drupal as an extensible library platform". Library hi tech, v. 27, n. 2, pp. 252-267. http://dx.doi.org/10.1108/07378830910968209

Moore, Tony A. (2008). "Assessing and adapting Drupal content management system as a digital library application". Procs of the American Society for Information Science and Technology, v. 45, n. 1, pp. 1-4.

http://dx.doi.org/10.1002/meet.2008.14504503131

Rosenthal, Danielle; Bernardo, Mario (2010). “Creating a library database search using Drupal". Code4Lib journal, v. 10. http://journal.code4lib.org/articles/2920

Subirats, Imma; Malapela, Thembani; Dister, Sarah; Zeng, Marcia; Goovaerts, Marc; Pesce, Valeria; Jaques, Yves; Anibaldi, Stefano; Keizer, Johannes (2012). "Reorienting open repositories to the challenges of the semantic web: experiences from FAO's contribution to the resource processing and discovery cycle in repositories in the agricultural domain". $6^{\text {th }}$ Research conf on metadata and semantics research, MTSR 2012, Communications in computer and information science, v. 343, pp. 158-167.

http://eprints.rclis.org/17733

Tramullas, Jesús (ed.) (2010). Drupal para bibliotecas y archivos. Zaragoza: Fund. Zaragoza Ciudad del Conocimiento. ISBN: 9784461396115 http://eprints.rclis.org/14400

Tramullas, Jesús; Garrido-Picazo, Piedad (2010). “Drupal: módulos para bibliotecas, archivos y servicios de información y documentación." En: Tramullas, Jesús (ed.) Drupal para bibliotecas y archivos. Zaragoza: Fund. Zaragoza Ciudad del Conocimiento, pp. 21-28. ISBN: 9784461396115

Vacek, Rachel; Watkins, Sean; Morris, Christina M.; Keller, Derek (2010). "Improving the Drupal user experience". Code4Lib journal, v. 12.

http://journal.code4lib.org/articles/4578

Varnum, Ken J. (2012). Drupal in libraries. Chicago: ALA TechSource y Neal-Schuman. ISBN: 9781555707781 